Wayne State University

\title{
DigitalCommons@WayneState
}

Library Scholarly Publications

Wayne State University Libraries

$1-1-2008$

\section{Making Sense of Dietary Supplements: The Dietary Supplements Labels Database}

Annette M. Healy

WayneState University, amhealy@wayne.edu

\section{Recommended Citation}

Healy, Annette M., "Making Sense of Dietary Supplements: The Dietary Supplements Labels Database" (2008). Library Scholarly Publications. Paper 33.

http://digitalcommons.wayne.edu/libsp/33 


\title{
HEALTH SITINGS
}

\section{Lynda M. Baker, Column Editor}

\section{Making Sense of Dietary Supplements: \\ The Dietary Supplements Labels Database \\ <http://dietarysupplements.nlm.nih.gov/> \\ Annette M. Healy}

\begin{abstract}
This article describes the Dietary Supplements Labels Database, a new resource from the National Library of Medicine that integrates information from dietary supplement manufacturers, government agencies, and clinical research into an easy-to-use interface. This database contains information on more than 2,000 brands of dietary supplement and more than 800 active ingredients. This resource will greatly assist consumers and health care providers in evaluating the safety, efficacy, and quality of dietary supplements. Features of the resource will be described as well as the browse and search methods used to access the information.
\end{abstract}

KEYWORDS. Dietary supplements, consumer health information, vitamins, minerals, herbs, databases

AUTHOR. Annette M. Healy, MLIS, PhD (amhealy@wayne.edu) is Information Services Librarian, Science \& Engineering Library, Wayne State University, Detroit, MI 48202.

Comments and suggestions should be sent to the column editor: Lynda M. Baker (aa0838@wayne.edu) 


\section{INTRODUCTION}

Dietary supplement use is increasingly popular in the United States. Sales of dietary supplements have grown from $\$ 4$ billion in 1994 to $\$ 21$ billion today. ${ }^{1}$ Dietary supplements are regulated by the U.S. Food and Drug Administration (FDA) but are not subject to the same regulations as prescription and over-the-counter medications. The Dietary Supplement Health and Education Act (DSHEA) of 1994 established a new category for dietary supplements and created a new regulatory framework governing the safety and labeling of these products. ${ }^{2}$ Under DSHEA, a dietary supplement, defined as a product intended to supplement the diet, contains vitamins, minerals, herbs, and/or amino acids; is taken in tablet, capsule, powder, or liquid form; and is labeled on the front panel as a dietary supplement. ${ }^{3}$

The enactment of DSHEA decreased barriers to dietary supplements but has also raised some safety and efficacy issues. ${ }^{2}$ For example, manufacturers of dietary supplements are not required to provide evidence of safety and efficacy before a product is marketed. Also, FDA regulations have not been established to ensure the purity, quality, or strength of dietary supplements, and there are no guidelines that limit the serving size or amount of a nutrient in a dietary supplement. In addition, once a dietary supplement is on the market, the FDA must demonstrate that the product is unsafe before it can take regulatory action. Thus, for consumers to make informed decisions regarding dietary supplement use, they require access to information on their safety, quality, and efficacy.

Current law allows manufacturers to make claims about dietary supplements and their

relationship to health. ${ }^{4}$ Only substances classified as drugs can include claims that they diagnose, treat, cure, or prevent disease; but dietary supplement manufacturers can make health claims that 
describe a relationship between an active ingredient and reducing the risk of disease or condition (e.g., calcium reduces the risk of osteoporosis). This type of claim requires authorization from the FDA based on significant scientific evidence. Manufacturers may also make structure/function claims that associate an ingredient with normal structure or function (e.g., calcium builds strong bones). This type of claim requires that the manufacturer be able to substantiate that the statement is truthful but does not require FDA review or approval. The subtle differences between claims made by drug manufacturers and dietary supplement manufacturers may lead to confusion on the part of the consumer about the effectiveness and uses of a given supplement.

Given the laws that govern the manufacture, marketing, and labeling of dietary supplements, consumers will benefit from having access to several types of information when considering a dietary supplement. For effective evaluation, consumers need access to information regarding the safety, efficacy, appropriate dose, and the quality of a dietary supplement. In addition, consumers with dietary restrictions will want information about other ingredients in the supplement that may affect their health. Locating this type of information can be a time-consuming process that involves viewing supplement labels, manufacturers' Web sites, government Web sites, consumer health Web sites, and the medical literature. The Dietary Supplements Labels Database is a new resource that streamlines this process by providing access to a wide range of information gathered from manufacturers, government agencies, and clinical research studies in a single Web site. This database, which is freely available on the Internet, will also be a useful tool for health care providers when assisting patients in evaluating the risks and benefits associated with specific supplements.

\section{ABOUT THE DIETARY SUPPLEMENTS LABELS DATABASE}

The Dietary Supplements Labels Database <http://dietarysupplements.nlm.nih.gov/> was 
launched in October 2007 by the National Library of Medicine (NLM). As of January 2008, the database contained information on over 2,000 brands of dietary supplements sold in the United States, as well as 877 active ingredients contained in those brands and the 150 manufacturers that produce those brands. Products in the database were selected by the database provider, DeLima Associates, based on market share and shelf presence in retail stores. The information in the database is collected from a variety of publicly available sources, including brand-specific labels and information from manufacturers' Web sites. Not all products from a given brand are in the database, but products will continue to be added as information becomes available. Information in the database enables users to determine what ingredients are in a specific brand, to compare ingredients in different brands, and to evaluate health claims made by manufacturers. The database includes authoritative information on specific ingredients in dietary supplements, including research studies that evaluate their uses, adverse effects, and mechanisms of action. By comparing health claims and the clinical evidence that support those claims, health care providers and consumers can better understand the risks and benefits of dietary supplement use. The database also provides convenient access to warnings and recalls of dietary supplements from the FDA. Thus, the Dietary Supplements Labels Database is a unique easy-to-use resource that combines information from manufacturers, government agencies, and clinical research studies in a single location to assist consumers in making informed decisions regarding dietary supplement use.

\section{FINDING INFORMATION}

The Dietary Supplements Labels Database is well organized and easy to navigate. The home page (see Figure 1) provides basic information on the purpose of the database and the types of information it contains. A black navigation bar located at the top of each page includes clearly 
labeled links to useful resources such as Frequently Asked Questions (FAQ), Glossary, and Related Resources (described later in this article). A Quick Search box is prominently located at the top of the page that can be used for brand name, active ingredient, or manufacturer searches. In the left column, there are three Browse \& Search options that will be described in detail later.

\section{[PLACE FIGURE 1 HERE]}

\section{Legend: FIGURE 1. Dietary Supplements Labels Database Home Page Reprinted with permission from the National Library of Medicine [place under figure]}

Links to Recalls and Warnings are also conveniently located on the home page for easy access. The link to Warnings provides information collected by MedWatch, the FDA Safety Information and Adverse Event Reporting Program. In January 2008, there were warnings available from 1997 to July 2007. An alphabetical list of products is located at the top of the page; each product is hyperlinked to more detailed information, including date of notice, summary of findings, actions taken, and links to announcements such as press releases. In January 2008, the link to Recalls provided information on recalls of dietary supplements from 2002 to 2007. For each year, a list of recalled products is provided and each product is hyperlinked to detailed recall information, including product name, lot numbers, where the product was distributed, and the reason for the recall. Having warning and recall information conveniently located together in an easily accessible format will greatly benefit consumers and health care providers that have questions regarding dietary supplement recalls and product warnings.

\section{Browse \& Search}


As noted earlier, there are three Browse \& Search options: brand, active ingredient, and manufacturer. For each option, there is a search box and an alphabetical list for browsing. Each alphabetical list has the letters of the alphabet arranged on separate pages, and links to each letter of the alphabet are prominently located at the top of each page so that users can quickly locate a brand, ingredient, or manufacturer. Browsing the alphabetical lists is a convenient way to locate an item of interest and is especially helpful when the user is unsure of the correct spelling or exact name. The Brand Search option includes an additional feature that allows users to limit their search by up to three of 32 possible dietary restrictions such as vegetarian, no gluten, or no sugar. Users who need to locate supplements that meet specific dietary restrictions will be able to quickly narrow their search to products that meet their needs. It should be noted that the Brand search box can also be used to search for an active ingredient that meets certain dietary restrictions. Finally, the Browse \& Search Brands option includes a third choice, Label Search. Users can search the text of each product label in the database for a keyword of interest such as a specific ingredient or a health condition. For example, a search for" arthritis" resulted in 28 product labels that contain the word "arthritis." Clicking on the name of a product reveals the product label information with the search term highlighted. This feature allows users to quickly identify all products that contain a specific ingredient or that may address a specific health issue.

\section{Working with Results}

Once a specific product is identified, clicking on its link brings the user to specific information on that product; this information is located under three tabs: Product Information, Ingredient Information, and Manufacturer Information. The Product Information tab includes the information available on the product label, including serving size, other ingredients, manufacturer's 
suggested use, label warnings, and claims made by the manufacturer (see Figure 2). At the top of each Product Information page the following disclaimer is prominently displayed to notify consumers of the limitations of dietary supplements: "The label claims have not been evaluated by the Food and Drug Administration. This product is not intended to diagnose, treat, cure or prevent any disease. Consult your healthcare professionals before taking any dietary supplements." If available, links are provided to fact sheets on the active ingredients; these fact sheets have been produced by the National Institutes of Health (NIH) or other authoritative sources. Also if available, information is provided on third-party testing of the product. Several organizations provide independent testing of supplements, and the organizations included in this database are described in the glossary. These tests generally verify the identity and quantity of the active ingredients and look for the presence of possible contaminants. Access to independent test results greatly assists consumers in evaluating different brands of supplements.

\section{[PLACE FIGURE 2 HERE]}

\section{Legend: FIGURE 2. Product Information for Caltrate 600}

\section{Reprinted with permission from the National Library of Medicine [place under figure]}

The Ingredient Information tab identifies each active ingredient in a product, the amount contained, and the percent of the daily value in each serving. Each active ingredient is linked to detailed information from selected non-manufacturer sources such as government agencies and clinical research studies (see Figure 3). This active ingredient information can also be found by using the Browse \& Search Active Ingredients option located on the home page. Information included on an active ingredient includes a Description, Related Names, and Reference Links. The amount of information available under Reference Links will vary depending on what is known 
about the ingredient. Reference Links are divided into four categories: General Information, Uses, Adverse Effects, and Mechanism of Action. General Information includes Fact Sheets written for consumers from MedlinePlus, the Office of Dietary Supplements, the National Center for Complimentary and Alternative Medicine, and the National Cancer Institute. Clinical and research studies on the active ingredient are arranged under the categories Uses, Adverse Effects, and Mechanism of Action. Information under Uses includes links to Clinical Trials of the ingredient from NLM's ClincialTrials.gov; citations of articles in NLM's PubMed that report on clinical trials testing the ingredient; effectiveness literature that includes citations of reviews and case reports from PubMed on the therapeutic use of the ingredient; and administration literature that includes citations to articles from PubMed on the administration and dosage of the ingredient. For information on Adverse Effects, citations to articles in PubMed are included that report on adverse effects or poisoning by the active ingredient. For information on the Mechanism of Action, citations to articles from PubMed on the actions and kinetics of the active ingredient are listed. Each list of citations from PubMed is the result of a predefined search on the subject that will be updated as new studies are published that meet the search criteria.

\section{[PLACE FIRGURE 3 HERE]}

\section{Legend: FIGURE 3. Active Ingredient Information for Thiamin}

\section{Reprinted with permission from the National Library of Medicine [place under figure]}

At this time, information on some active ingredients is limited, and fact sheets containing synthesized information written for consumers for all of the active ingredients in the database are not available. For those ingredients without fact sheets, users must rely on the medical literature for information on uses and adverse effects. In addition, for some active ingredients, coverage in the 
medical literature is also limited. Providing links to PubMed searches is an efficient method for supplying current information on active ingredients. These PubMed searches will be most helpful to health care professionals who possess the background necessary for understanding and evaluating the information. In addition, they are likely to be familiar with PubMed and have access to full-text articles. For consumers, the provision of PubMed searches presents a number of barriers to information on active ingredients. Consumers will likely be unfamiliar with PubMed, literature searches, and interpreting citations. In addition, many consumers will not have access to the cited articles, and those that do access articles may not be able to understand or interpret them because of the advanced reading level and the use of scientific terminology and methodology. For these users, access to information on active ingredients may require interpretation by a family member, health care provider, or information professional.

The Manufacturer Information tab provides contact information for the manufacturer, including address, phone number, and URL, as well as a link to all brands by the manufacturer. This information is provided so that consumers may contact the manufacturer with questions about a product. The same manufacturer information can also be located by using the Browse \& Search Manufacturer option located on the home page.

\section{ADDITIONAL FEATURES}

The Dietary Supplements Labels Database provides additional features that enhance its usefulness. Links to these features are located in the black navigation bar at the top of each page. The Glossary provides definitions for terms and abbreviations that may be unfamiliar to users. The Help section is nicely organized with descriptions and illustrations of how to browse and search brands, active ingredients, and manufacturers. The FAQ provides answers to questions regarding 
what dietary supplements are, who regulates them, and who validates their safety and efficacy. The Related Resources tab provides links to general information about dietary supplements written for consumers and produced by federal agencies as well as links to FDA recalls and warnings. Easy access to these resources will help consumers become more knowledgeable about dietary supplements and their safe use. It also provides health care providers with a resource for educational materials for their patients

\section{CONCLUSION}

The dietary supplement market has exploded in recent years, and consumers can choose from numerous brands, active ingredients, and health claims. Previously, consumers interested in making informed decisions about dietary supplements would have to consult several resources to locate the needed information. The Dietary Supplements Labels Database brings together resources from supplement manufacturers, government agencies, and clinical studies into an easy-to-use Web interface that is freely accessible on the Internet. Using the Dietary Supplements Labels Database, users can conduct numerous tasks at the same Web site, including comparing brands of supplements, identifying supplements that have passed third-party testing, identifying supplements that meet dietary restrictions, quickly locating FDA warnings and recalls, and identifying research articles that contain clinical data regarding the use or adverse effects of an active ingredient. A welcome enhancement to the database would be the provision of a link to a printable version of information found in the Dietary Supplements Labels Database. Currently, for some users, printing in the portrait orientation does not capture all the information on a Web page.

Current knowledge about dietary supplements is incomplete and rapidly changing. The Dietary Supplements Labels Database strives to provide the latest information available. Because 
research on dietary supplements is ongoing, it is understandable that information available for active ingredients varies in quantity and accessibility. As the landscape of information on dietary supplements changes, NLM will be able to broaden the depth and breadth of information available in the database. The Dietary Supplements Labels Database is a welcome resource that greatly enhances the ability of consumers and health care providers to locate information on dietary supplements and to use that information to make informed decisions.

\section{REFERENCES}

1. Saldanha, L. “The Dietary Supplement Marketplace: Constantly Evolving.” Nutrition Today 42(March/April 2007): 52-4.

2. Larsen, L.L., and Berry, J.A. “The Regulation of Dietary Supplements.” Journal of the American Academy of Nurse Practitioners 15(September 2003): 410-4.

3. National Institutes of Health. Office of Dietary Supplements. "Dietary Supplements: Background Information.” (April 2006). Available: <http://ods.od.nih.gov/factsheets/DietarySupplements.asp>. Accessed: December 11, 2007.

4. Turner, R.E.; Degnan, F.H.; and Archer, D.L. "Label Claims for Foods and Supplements: A Review of the Regulations." Nutrition in Clinical Practice 20(February 2005): 21-32. 